\title{
Helicobacter pylori infection over bile reflux: No influence on the severity of endoscopic or premalignant gastric lesion development
}

\author{
ANDREEA SZŐKE $^{1 *}$, SIMONA MOCAN $^{2}$ and ANCA NEGOVAN ${ }^{3 *}$ \\ ${ }^{1}$ Department of Pathology, Mureș County Clinical Hospital, 540061 Târgu Mureș; ${ }^{2}$ Department of Pathology, \\ Emergency County Hospital, 540136 Târgu Mureș; ${ }^{3}$ Department of Clinical Science-Internal Medicine, \\ 'George Emil Palade' University of Medicine, Pharmacy, Science, and Technology, 540139 Târgu Mureș, Romania
}

Received March 12, 2021; Accepted April 12, 2021

DOI: $10.3892 / \mathrm{etm} .2021 .10198$

\begin{abstract}
Helicobacter (H.) pylori infection and duodenogastric reflux (DGR) are both linked to endoscopic and premalignant gastric lesion development, but it is still unclear whether they are independent or have a causal relationship. This study investigated the histologic gastric changes in patients with primary DGR and H. pylori infection, as well as their endoscopic findings, symptoms, drug consumption, and social behavior in comparison with patients presenting only DGR. The study included 560 patients with primary DGR on endoscopy divided into two groups, according to the presence/absence of $H$. pylori infection on biopsy (utilizing usual stainings and immunohistochemical methods). There was no significant difference in terms of age and sex, nor in the frequency of diabetes or esophagitis between the studied groups. Epigastric pain was associated with $H$. pylori-positive biopsies in multivariate logistic regression analysis $(\mathrm{P}=0.005)$. Although without statistical significance, severe endoscopic lesions and premalignant gastric lesions were more frequent in the $H$. pylori group ( 45.1 vs. $28.4 \%$ and 37.4 vs. $32.3 \%$, respectively). In patients with DGR, the final multivariate model revealed a positive association between smoking and immunohistochemically confirmed $H$. pylori infection ( $\mathrm{P}=0.02, \mathrm{OR}=1.88,95 \%$ confidence intervals $(\mathrm{CI})=1.10$ to 3.21$)$, but a negative effect of proton pump inhibitor consumption $(\mathrm{P}<0.001, \mathrm{OR}=0.50$, $95 \% \mathrm{CI}=0.35$ to 0.73 ). In conclusion, in patients with $H$. pylori infection and DGR, epigastric pain was the main reason for the endoscopic investigation. H. pylori infection over DGR
\end{abstract}

Correspondence to: Dr Anca Negovan, Department of Clinical Science-Internal Medicine, 'George Emil Palade' University of Medicine, Pharmacy, Science, and Technology, 38 Gheorghe Marinescu Street, 540139 Târgu Mureș, Romania

E-mail: ancanegovan@yahoo.com; anca.negovan@umfst.ro

*Contributed equally

Key words: bile reflux, Helicobacter pylori, smoking, intestinal metaplasia, atrophic gastritis, endoscopy did not influence the severity of endoscopic or premalignant gastric lesion development. Furthermore, smoking is directly related to immunohistochemically assessed active $H$. pylori infection in patients with bile reflux.

\section{Introduction}

Duodenogastric reflux (DGR, bile reflux) consists of a retrograde passage of alkaline duodenal and pancreatic content into the stomach, which can lead to mucous barrier disruption and direct chemical damage to the surface gastric epithelium. DGR is common after gastric surgery, cholecystectomy and pyloroplasty, but it can also occur due to antroduodenal motility disorder (primary DGR) $(1,2)$. Primary bile reflux does not have a standardized diagnosis. If an upper digestive endoscopy (UDE) reveals a large amount of bile in the stomach, mucosal fragility, erythema/erosions/ulcers in a patient with epigastric pain, nausea/vomiting, the diagnosis is usually DGR (3).

Gastric cancer (GC), particularly intestinal-type, arises via the carcinogenic gastric cascade initiated by Helicobacter $(H$.$) pylori and involves several histological$ changes: Active inflammation, non-atrophic chronic gastritis, multifocal atrophic gastritis (AG), intestinal metaplasia (IM), dysplasia, and cancer (4-6). A previous study demonstrated an increased risk of IM in patients with high levels of bile acid reflux, hence DGR might contribute to the development of precancerous lesions and GC (7).

The results of studies investigating the relationship between $H$. pylori and bile reflux are contradictory. Some studies report that $H$. pylori colonization decreases as a result of high levels of bile acids (8), while others revealed a positive relationship between $H$.pylori and DGR, where DGR was found to increase the presence of bacteria $(9,10)$.

The present study questioned the importance of concomitant active $H$. pylori infection on endoscopic and histologic gastric changes in patients with biliary aggression. The objective was to investigate their association with possible demographic and clinical predictors (digestive symptoms, drug consumption, social habits, comorbidities) in order to identify potential strategies to limit progression toward stomach cancer, in a population with a high prevalence of long-standing H. pylori infection. 


\section{Patients and methods}

Subjects and data collection. A total of 2,014 patients with dyspeptic complaints or anemia, hospitalized at the Medical Clinic No. 2 in Târgu Mureș Emergency County Hospital, Romania, underwent an upper digestive endoscopy (UDE) between January 2016 and December 2019. The present study involved those patients diagnosed with DGR following UDE $(\mathrm{n}=560)$ divided into two groups: Study group (Bile reflux + H. pylori), 195 patients with $H$. pylori infection confirmed upon biopsy specimens utilizing usual histochemical stains or immunohistochemical methods; and Control group (bile reflux), 365 patients negative for $H$. pylori infection on all biopsy sites or immunohistochemistry when performed.

Bile reflux diagnosis was based on detection of DGR and a large bile pool in the stomach during the endoscopic examination, in patients with a fasting period of $>10 \mathrm{~h}$.

Five random biopsy specimens were taken and examined for each patient: Two from the antrum, two from the corpus (the greater and the lesser curvature), and one from incisura angularis. The tissue samples were fixed in formalin, embedded in paraffin, and stained with routine hematoxylin and eosin, PAS-Alcian blue and modified Giemsa, followed by microscopic evaluation. H. pylori infection was considered negative if the bacteria were absent from all biopsy sites and positive if identified in at least one biopsy sample.

When no organism was detected using histochemical stains, but a high suspicion of infection existed due to persistent inflammation, particularly for patients with proton-pump inhibitor (PPI) therapy, the pathologist performed immunohistochemistry staining. The immunohistochemical staining was performed with FLEX polyclonal rabbit anti-Helicobacter pylori antibody, Ready to Use (Dako Autostainer) and using EnVision FLEX, High $\mathrm{pH}$ as a visualization system (Fig. 1).

In the database were noted the clinical examination, drug exposure, comorbidities, symptoms, and social habits of the patients. Exclusion criteria were acute bleeding episodes, previous gastrectomy or $H$. pylori eradication therapy, dysplasia, gastric/esophageal neoplasms, and missing data (drug exposure, social habits, biopsies).

Recorded digestive symptoms included epigastric pain, heartburn, flatulence, and nausea/vomiting. We considered chronic proton pump inhibitor therapy (pantoprazole, esomeprazole, omeprazole) if administered at regular doses for $\geq 3$ months before investigation.

Anemia and diabetes mellitus were registered when the diagnosis was made based on international clinical guideline criteria. For anemia, the cut-off values of hemoglobin levels according to the WHO definitions were: $12 \mathrm{~g} / \mathrm{dl}(7.5 \mathrm{mmol} / \mathrm{l})$ in women and $<13 \mathrm{~g} / \mathrm{dl}(<8.1 \mathrm{mmol} / \mathrm{l})$ in men (11). We considered alcohol consumption if patients declared a consumption of $\geq 10$ units (10 ml) of pure alcohol weekly. Patients registered as smokers were those that smoked $>5$ cigarettes/day, including quitters during the past 5 years.

Additional endoscopic and histologic aspects. During the endoscopic examination of the stomach the following data were recorded: Mucosal fragility, hyperemia, edema, petechiae/erosions/ulcers, hiatal hernia, and bile reflux. In order to describe the endoscopic appearance of reflux esophagitis and grade its severity, Los Angeles classification system was utilized (12). In the present study, esophagitis was recorded as present or absent.

We assessed gastro-duodenal lesions using a modified Lanza score: Grade 0, no mucosal lesions; Grade 1, one erosion (mucosal defect $<5 \mathrm{~mm}$ ) or petechiae (hemorrhagic area without mucosal defect); Grade 2, 2-10 erosions/petechiae; Grade 3,>10 erosions/petechiae; Grade 4, gastric ulcer (defect $>5 \mathrm{~mm}$ in diameter). Patients having Lanza scores between 0 and 2 were registered with no/mild endoscopic lesions, and those with scores of 3 and 4 were diagnosed with severe endoscopic lesions (13).

Patients with foveolar hyperplasia, vascular dilation of superficial mucosal capillaries, fibromuscular replacement of the lamina propria and without inflammation were diagnosed with reactive gastropathy.

Atrophy was registered as the loss of normal glands in both the antrum/corpus. IM has various aspects on histology. The pathologist considered complete IM when some parts of the gastric epithelium had a small intestinal epithelium aspect, with eosinophilic enterocytes, goblet cells and brush border. Incomplete IM was defined as colonic epithelium in gastric mucosa, containing mucin droplets with irregular form and different sizes, as well as the absence of the brush border. The Updated Sydney System was used to assess the intensity of mononuclear inflammatory cellular infiltrates and inflammatory activity (neutrophilic infiltrations) in the diagnosis of gastritis. The degree of the atrophy and IM was defined using the OLGA/OLGIM (Operative Link on Gastritis/IM Assessment) (14). In addition, patients with antral-type glands in the body of the stomach were diagnosed with pseudopyloric metaplasia.

Statistical analysis. For statistical analysis, JASP (JASP graphical program for statistical analysis supported by the University of Amsterdam) program v.0.12.1 (https://jasp-stats. org/) was applied. Mean \pm standard deviation or absolute (relative) frequencies were used to summarize the demographic and clinical data. To identify significant differences in terms of quantitative demographic characteristics, Mann Whitney U test was applied, while for comparison of categorical variables the Chi-square or Fisher's exact test were utilized, both followed by univariate logistic regression analysis. All significant factors and factors whose unadjusted estimated significance level was $\mathrm{P}<0.25$ in univariate analysis were selected as candidates for multivariate logistic regression. The partial likelihood ratio test was applied to decide the analyzed variables in the final model. The odds ratio (OR) and 95\% CI quantified the magnitude of the association. A two-sided P-value $<0.05$ was considered statistically significant in all tests.

\section{Results}

The observed relative frequency of $H$. pylori infection among patients with bile reflux was $34.82 \%$. There was no significant difference in terms of age between the Study and Control groups: Median (Q1-Q3) 62 years (52-72) for controls vs. 60 years (50.5-69.5) for cases, $\mathrm{U}=38206, \mathrm{P}=0.151$, 
Table I. Group differences regarding the studied factors in patients with DGR $\pm H$. pylori infection.

\begin{tabular}{|c|c|c|c|c|c|}
\hline \multirow[b]{2}{*}{ Variables } & \multicolumn{2}{|c|}{$\begin{array}{l}\text { Bile reflux }+H . \text { pylori } \\
\text { (study group) } \\
\left(\mathrm{n}_{1}=195\right)\end{array}$} & \multicolumn{2}{|c|}{$\begin{array}{c}\text { Bile reflux } \\
\text { (control group) } \\
\left(\mathrm{n}_{2}=365\right)\end{array}$} & \multirow[b]{2}{*}{ P-value } \\
\hline & No. & $\%$ & No. & $\%$ & \\
\hline Age $>65$ years & 65 & 33.3 & 150 & 41 & 0.0800 \\
\hline Female sex & 98 & 50.3 & 199 & 54.6 & 0.3700 \\
\hline Premalignant gastric lesions & 73 & 37.4 & 118 & 32.3 & 0.2200 \\
\hline Severe endoscopic lesions & 64 & 45.1 & 104 & 28.4 & 0.2800 \\
\hline Anemia & 32 & 16.4 & 67 & 18.3 & 0.6400 \\
\hline Esophagitis & 43 & 22.0 & 72 & 19.7 & 0.5100 \\
\hline Diabetes & 34 & 17.4 & 62 & 16.9 & 0.9000 \\
\hline Heartburn & 58 & 29.7 & 98 & 26.8 & 0.4800 \\
\hline Epigastric pain & 103 & 52.8 & 156 & 42.7 & 0.0261 \\
\hline Nausea/Vomiting & 33 & 16.9 & 55 & 15.0 & 0.6200 \\
\hline Flatulence & 50 & 25.6 & 67 & 18.3 & 0.0496 \\
\hline Smoking $^{\mathrm{b}}$ & 31 & 15.8 & 34 & 9.3 & 0.0263 \\
\hline Alcohol consumption $^{c}$ & 40 & 20.5 & 54 & 14.7 & 0.0900 \\
\hline PPI therapy & 73 & 37.4 & 185 & 50.6 & 0.0033 \\
\hline
\end{tabular}

${ }^{\mathrm{a}}$ Obtained from Chi-square or Fisher's exact tests; ${ }^{\mathrm{b}}$ Over 5 cigarettes/day; ${ }^{\mathrm{c}}$ More than 2 units/day, 1 unit=10 ml pure alcohol. $\mathrm{H}$. pylori, Helicobacter pylori; DGR, duodenogastric reflux; PPI, proton-pump inhibitor; OR, odds ratio; CI, confidence interval. P-values indicative of a significant result are presented in bold print.

A

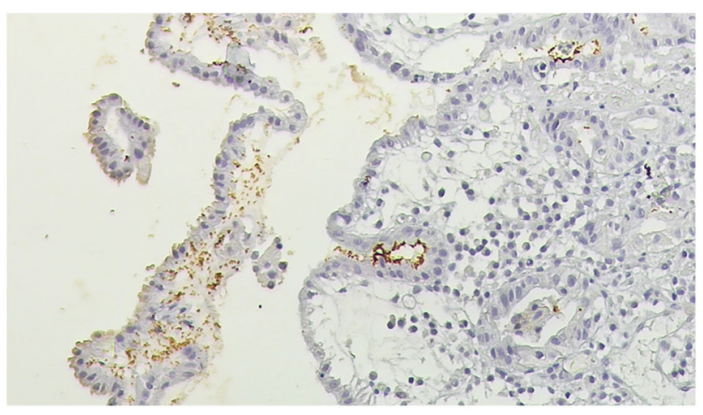

B

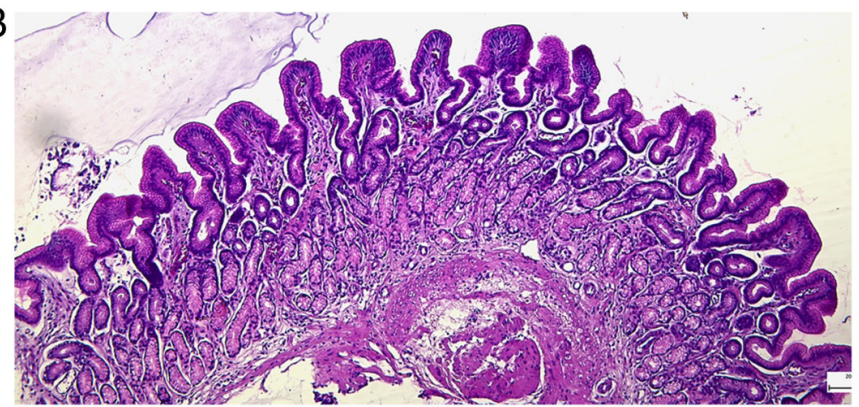

Figure 1. Histologic aspects in Helicobacter pylori gastritis compared to reactive gastropathy. (A) Immunohistochemical staining for Helicobacter pylori: With numerous positive microorganisms attached to the luminal surface of the foveolar cells; magnification, x10. (B) Reactive gastropathy: Foveolar hyperplasia with mild changes in the lamina propria, but without any inflammation. Hematoxylin and eosin staining; magnification, $\mathrm{x} 4$.

but patients $>65$ years, although without a statistically significant level, were more frequent in the Bile reflux group (without H. pylori infection). The differences between demographic and clinical characteristics are presented in Table I. Severe endoscopic lesions (score 3 and 4) and premalignant gastric lesions (IM and AG) were more common in patients with DGR reflux and $H$.pylori infection $[45.1 \%$ (64) vs. $28.4 \%$ (104) and $37.4 \%$ (73) vs. $32.3 \%$ (118), respectively], but without a statistically significant association (Table I).

In the Bile reflux group, the stomach histology most frequently demonstrated reactive gastropathy (44.4\% patients), followed by inactive chronic gastritis $(40.5 \%)$. Also, $15.1 \%$ of the patients were without histologic lesions.

The distribution of premalignant gastric lesions was similar between the Study and Control groups. Patients presented more frequent both IM and AG, but there were $41 \%$ cases and $39 \%$ controls of IM alone. AG, without IM, was found only in a small percentage of patients with premalignant gastric lesions (Table II).

In the univariate logistic regression model, smoking was a significant factor associated with DGR and $H$.pylori infection, while pre-treatment with PPI had a negative effect. Regarding the symptomatology, epigastric pain and flatulence were significant factors associated with $H$. pylori infection and bile reflux (Table III).

According to the multivariate logistic analysis results, in patients with DGR, smoking was significantly associated with $H$. pylori infection, while PPI therapy remained negatively associated with the immunohistochemically confirmed infection. The odds of H. pylori infection increased by 1.88 
Table II. Premalignant gastric lesion distribution.

\begin{tabular}{|c|c|c|c|c|c|c|c|}
\hline \multirow[b]{2}{*}{ Premalignant gastric lesions $(n=191)$} & \multicolumn{2}{|c|}{$\begin{array}{c}\text { Bile reflux }+ \\
\text { H. pylori } \\
\text { (Study group) } \\
\quad\left(\mathrm{n}_{1}=73\right)\end{array}$} & \multicolumn{2}{|c|}{$\begin{array}{l}\text { Bile reflux } \\
\text { (Control } \\
\text { group) } \\
\left(\mathrm{n}_{2}=118\right)\end{array}$} & \multirow[b]{2}{*}{ P-value ${ }^{a}$} & \multirow[b]{2}{*}{ OR } & \multirow[b]{2}{*}{$95 \%$ CI } \\
\hline & No. & $\%$ & No. & $\%$ & & & \\
\hline IM & 30 & 41.1 & 46 & 39 & 0.80 & 1.07 & 0.65 to 1.75 \\
\hline $\mathrm{AG}$ & 2 & 2.7 & 8 & 6.8 & 0.32 & 0.38 & 0.07 to 1.87 \\
\hline $\mathrm{IM}+\mathrm{AG}$ & 41 & 56.2 & 64 & 54.2 & 0.88 & 1.08 & 0.60 to 1.94 \\
\hline
\end{tabular}

${ }^{\mathrm{a}}$ Obtained from Chi-square test or Fisher's exact test. H. pylori, Helicobacter pylori; IM, intestinal metaplasia; AG, atrophic gastritis; OR, odds ratio; CI, confidence interval.

Table III. Results from the univariate binary logistic regression.

\begin{tabular}{|c|c|c|c|c|}
\hline Variable & Statistics Z & P-value ${ }^{a}$ & Crude OR & $95 \% \mathrm{CI}$ \\
\hline Age $>65$ years & -1.79 & 0.07 & 0.71 & 0.49 to 1.03 \\
\hline Female sex & -0.96 & 0.33 & 0.84 & 0.59 to 1.19 \\
\hline Severe endoscopic lesions & 1.06 & 0.28 & 1.22 & 0.84 to 1.78 \\
\hline Anemia & -0.57 & 0.56 & 0.87 & 0.54 to 1.38 \\
\hline Esophagitis & 0.64 & 0.51 & 1.15 & 0.75 to 1.76 \\
\hline Diabetes & 0.13 & 0.89 & 1.03 & 0.65 to 1.63 \\
\hline Heartburn & 0.72 & 0.46 & 1.15 & 0.78 to 1.69 \\
\hline Epigastric pain & 2.27 & 0.023 & 1.50 & 1.05 to 2.12 \\
\hline Nausea/Vomiting & 0.57 & 0.56 & 1.14 & 0.71 to 1.84 \\
\hline Flatulence & 2.09 & 0.036 & 1.56 & 1.02 to 2.37 \\
\hline Smoking $^{\mathrm{b}}$ & 2.29 & 0.022 & 1.84 & 1.09 to 3.10 \\
\hline Alcohol consumption ${ }^{c}$ & 1.17 & 0.08 & 1.48 & 0.94 to 2.33 \\
\hline PPI therapy & -2.98 & 0.003 & 0.58 & 0.40 to 0.83 \\
\hline
\end{tabular}

${ }^{a}$ Crude P-values obtained from Wald's test; Response variable: Presence of Helicobacter $(H$.$) pylori infection in patients with biliary reflux.$ ${ }^{\mathrm{b}}$ Over 5 cigarettes/day; ${ }^{\mathrm{C}}$ More than 2 units/day, 1 unit: $10 \mathrm{ml}$ pure alcohol. Crude OR, unadjusted odds ratio; CI, confidence interval; PPI therapy, proton-pump inhibitors. P-values indicative of a significant result are presented in bold print.

Table IV. Final multivariable logistic regression model.

\begin{tabular}{|c|c|c|c|c|c|}
\hline Variables & $b^{a}$ & SE & P-value ${ }^{b}$ & Adjusted OR & $95 \% \mathrm{CI}$ \\
\hline Smoking & 0.63 & 0.27 & 0.020 & 1.88 & 1.10 to 3.21 \\
\hline PPI therapy & -0.67 & 0.19 & $<0.001$ & 0.50 & 0.35 to 0.73 \\
\hline Flatulence & 0.36 & 0.21 & 0.090 & 1.44 & 0.93 to 2.21 \\
\hline Epigastric pain & 0.52 & 0.18 & 0.005 & 1.69 & 1.17 to 2.45 \\
\hline Intercept & -0.73 & 0.14 & $<0.001$ & 0.47 & 0.35 to 0.64 \\
\hline
\end{tabular}

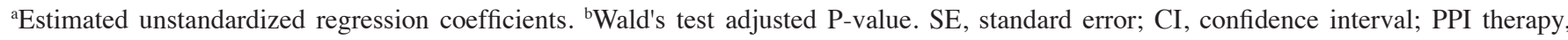
proton-pump inhibitors. P-values indicative of a significant result are presented in bold print.

(95\% CI 1.10 to 3.21 ) in smokers with bile reflux, effect adjusted for other covariates. Epigastric pain was related to $H$. pylori-positive patients, while flatulence had only a tendency towards statistical significance $(\mathrm{P}=0.09)$ (Table IV).
The final multivariable logistic regression model was statistically significant, $\chi^{2}(555)=26.52, \mathrm{P}<0.001$. The model correctly classified $66 \%$ of patients and the Brier score of 0.21 indicates a good accuracy of the model. 


\section{Discussion}

The diagnosis and clinical consequences of DGR are challenging, particularly for patients without known risk factors (gastric surgery) (15). High amounts of bile acids can cause direct gastric mucosal injury by its components, triggering inflammation (16). Because bile reflux and $H$. pylori often coexist and both are involved in the pathogenesis of gastritis, research has examined the relationship between these two entities. All patients included in this study had endoscopic criteria for biliary reflux and potential chemical aggression of the gastric mucosa. Only less than half of patients without active $H$. pylori infection (Control group) presented histologic features of gastropathy (44.4\%), while $15 \%$ had no histologic changes. The absence of histologic gastropathy supports less aggressive or recurrent exposure to bile acid or no biopsy from those areas with chemical aggression. In patients with active H. pylori infection, the chemical aggression is 'covered' by histologic inflammatory changes. Reexamination after eradication therapy may appreciate the role of each factor in the changes in gastric mucosa.

A recent article suggests a molecular mechanism related to the FXR/NF-кB signaling pathway, CDX2, and MUC2 implicated in IM occurrence and progression in patients with bile reflux (17). In addition, researchers have questioned the role of cytokines and their gene variants in premalignant gastric lesion development in patients with $H$. pylori infection (18). The risk of GC, mucosal aggression, symptoms and interaction with $H$.pylori infection are important issues in managing patients with DGR in daily practice as $H$. pylori favors the release of gastrin in the antrum, decreases peristaltic movements, and determines bile reflux (16). Although a higher prevalence of IM has been reported in patients with both DGR and $H$. pylori (10), in the studied population, H. pylori infection over bile reflux appeared not to exert significant additional influence on IM and AG development.

Severe endoscopic lesions were not significantly associated with concomitant $H$. pylori infection in the studied groups, sustaining previous findings in patients with gastric surgery in whom $H$. pylori did not play a substantial role in ulcer recurrence (19).

In the present study, epigastric pain was the most common complaint in both groups and was significantly associated with $H$. pylori infection, an effect that remained significant also in multivariate analysis. Another study investigating the symptoms in patients with $H$. pylori infection and bile reflux, reported nausea as the main complaint in patients with DGR, while in patients with $H$. pylori gastritis, epigastric pain was the most frequent symptom, followed by heartburn (20). Nevertheless, H. pylori infection was detected in only $40 \%$ of dyspeptic patients in the Romanian population, the role of other underlying conditions playing an important role (21).

The role of bile reflux in reflux esophagitis pathogenesis assumes chemical injury, but a recent study suggests that acidic bile salts induce esophagitis by a cytokine-mediated inflammation injury, driven by hypoxia-inducible factor $2 \alpha$ (HIF-2 $\alpha$ ) (22). Regarding the relationship between H.pylori and esophagitis, a meta-analysis showed that eradication of $H$. pylori increased the risk of esophagitis development, compared with patients without eradication. However, in the present study, the number of cases with esophagitis was similar between the two groups (23).

In the studied population, patients with DGR and negative for $H$. pylori infection were found to be consuming more frequent proton-pump inhibitors (PPIs). The effect of PPIs on decreasing the bacterial load in biopsy samples is well-known (24); therefore, to counteract the possible false-negative results when the suspicion of infection persisted due to inflammatory changes on histology, pathologists performed immunohistochemistry staining. High number of PPI prescriptions may be explained by the rich symptomatology attributed to both gastric acid secretion and DGR.

In the studied population, the multivariate logistic analysis revealed smoking is associated with $H$. pylori infection in patients with DGR. This result is in line with studies demonstrating that smoking affects gastro-duodenal motility and favors bile reflux into the stomach. Furthermore, smoking imbalances the antioxidant equilibrium and increases the risk of H.pylori infection (25).

The strength of the present study is the systematic histologic assessment of both antrum and corpus changes in patients with biliary reflux on endoscopy, tied with a thorough description of the most relevant clinical and endoscopic findings. Patients with a non-invasive diagnosis of $H$. pylori infection often complain about the persistence of symptoms after eradication therapy. Identifying associations between bile reflux and clinicopathological parameters may offer possible strategies to manage the symptoms and follow-up of this group of patients. The study has all the limitations of a retrospective observational study. The lack of bile reflux measurement and $\mathrm{pH}$ monitoring, and patients enrolled from the same geographical area are aspects to be improved.

In conclusion, in the studied population, $H$. pylori infection over DGR did not exert significant additional influence on the severity of endoscopic lesions, neither in premalignant gastric lesion development. Epigastric pain is more frequent in patients with bile reflux and $H$. pylori infection than in uninfected patients. Smoking was associated with $H$. pylori infection in patients with DGR, while $H$. pylori infection did not influence esophagitis occurrence. In patients with DGR diagnosis, PPI therapy had a negative association with immunohistochemically assessed $H$. pylori infection active gastritis.

\section{Acknowledgements}

Not applicable.

\section{Funding}

This research study was supported by an Internal Research Grant from the University of Medicine, Pharmacy, Sciences, and Technology of Târgu Mureș (no. 615/12/17.01.2019).

\section{Availability of data and materials}

The datasets analyzed during the current study are available from the corresponding author on request. 


\section{Authors' contributions}

Conceptualization of the study design was achieved by AN and AS. Methodology was the responsibility of AN. Formal analysis was conducted by AN and AS. Study investigation was conducted by AN and SM. Resources were obtained by AN and data curation was performed by AN and AS. Writing and original draft preparation was done by AS, AN and SM. Writing, review and editing was done by $\mathrm{AN}$ and AS. Supervision was conducted by $\mathrm{AN}$, and project administration and funding acquisition were the responsibility of AN. All authors have read and agreed to the published version of the manuscript.

\section{Ethics approval and consent to participate}

The research was approved by the Ethics Committee of the University of Medicine and Pharmacy of Târgu Mureș, Romania $(282,19.07 .2019)$. The patient informed consent was obtained for regular endoscopic investigation.

\section{Patient consent for publication}

Not applicable.

\section{Competing interests}

The authors state that they have no competing interests.

\section{References}

1. Tatsugami $\mathbf{M}$, Ito $\mathbf{M}$, Tanaka $\mathrm{S}$, Yoshihara $\mathbf{M}$, Matsui $\mathrm{H}$, Haruma K and Chayama K: Bile acid promotes intestinal metaplasia and gastric carcinogenesis. Cancer Epidemiol Biomarkers Prev 21: 2101-2107, 2012.

2. Agin M and Kayar Y: The effect of primary duodenogastric bile reflux on the presence and density of Helicobacter pylori and on gastritis in childhood. Medicina (Kaunas) 55: 775, 2019.

3. Chang WK, Lin CK, Chuan DC and Chao YC: Duodenogastric reflux: Proposed new endoscopic classification in sypmtomatic patients. J Med Sci 36: 1-5, 2016.

4. AmievaMandPeekRMJr:Pathobiologyof Helicobacterpylori-induced gastric cancer. Gastroenterology 150: 64-78, 2016.

5. González CA, Sanz-Anquela JM, Gisbert JP and Correa P: Utility of subtyping intestinal metaplasia as marker of gastric cancer risk. A review of the evidence. Int J Cancer 133: 1023-1032, 2013.

6. Jencks DS, Adam JD, Borum ML, Koh JM, Stephen S and Doman DB: Overview of current concepts in gastric intestinal metaplasia and gastric cancer. Gastroenterol Hepatol (N Y) 14 92-101, 2018.

7. Matsuhisa T, Arakawa T, Watanabe T, Tokutomi T, Sakurai K, Okamura S, Chono S, Kamada T, Sugiyama A, Fujimura Y, et al: Relation between bile acid reflux into the stomach and the risk of atrophic gastritis and intestinal metaplasia: A multicenter study of 2283 cases. Dig Endosc 25: 519-525, 2013.

8. Asfeldt AM, Steigen SE, Løchen ML, Straume B, Johnsen R, Bernersen B, Florholmen J and Paulssen EJ: The natural course of Helicobacter pylori infection on endoscopic findings in a population during 17 years of follow-up: The Sørreisa gastrointestinal disorder study. Eur J Epidemiol 24: 649-658, 2009.

9. Li D, Zhang J, Yao WZ, Zhang DL, Feng CC, He Q, Lv HH, Cao YP, Wang J, Qi Y, et al: The relationship between gastric cancer, its precancerous lesions and bile reflux: A retrospective study. J Dig Dis 21: 222-229, 2020.
10. Ladas SD, Katsogridakis J, Malamou H, Giannopoulou H, Kesse-Elia M and Raptis SA: Helicobacter pylori may induce bile reflux: Link between $H$. pylori and bile induced injury to gastric epithelium. Gut 38: 15-18, 1996.

11. Kasi $\mathrm{M}$ and Bowling T: Anaemia in gastroenterology. Medicine 43: 153-156, 2015.

12. Lundell LR, Dent J, Bennet JR, Blum AL, Armstrong D, Galmiche JP, Johnson F, Hongo M, Richter JE, Spechler SJ, et al: Endoscopic assessment of oesophagitis: Clinical and functional correlates and further validation of the Los Angeles classification. Gut 45: 172-180, 1999.

13. Lanza FL: Endoscopic studies of gastric and duodenal injury after the use of ibuprofen, aspirin, and other nonsteroidal anti-inflammatory agents. Am J Med 77: 19-24, 1984.

14. Capelle LG, de Vries AC, Haringsma J, Ter Borg F, de Vries RA, Bruno MJ, van Dekken H, Meijer J, van Grieken NC and Kuipers EJ: The staging of gastritis with the OLGA system by using intestinal metaplasia as an accurate alternative for atrophic gastritis. Gastrointest Endosc 71: 1150-1158, 2010.

15. McCabe ME IV and Dilly CK: New causes for the old problem of bile reflux gastritis. Clin Gastroenterol Hepatol 16: 1389-1392, 2018.

16. Huang H, Tian J, Xu X, Liang Q, Huang X, Lu J and Yao Y: A study on the roles of Helicobacter pylori in bile reflux gastritis and gastric cancer. J BUON 23: 659-664, 2018.

17. Yu JH, Zheng JB, Qi J, Yang K, Wu YH, Wang K, Wang CB and Sun XJ: Bile acids promote gastric intestinal metaplasia by upregulating CDX2 and MUC2 expression via the FXR/NF- $\mathrm{B}$ signalling pathway. Int J Oncol 54: 879-892, 2019.

18. Negovan A, Iancu M, Fülöp E and Bănescu C: Helicobacter pylori and cytokine gene variants as predictors of premalignant gastric lesions. World J Gastroenterol 25: 4105-4124, 2019.

19. Pantea M, Negovan A, Banescu C, Bataga S, Neagoe R, Mocan S and Iancu M: Factors associated with recurrent ulcers in patients with gastric surgery after more than 15 years: A cross-sectional single-center study. Gastroenterol Res Pract 2018: 8319481, 2018.

20. Barakat EAME, Abbas NF and El-Kholi NY: Primary bile reflux gastritis versus Helicobacter pylori gastritis: A comparative study. Egypt J Intern Med 30: 23-27, 2018.

21. Corojan AL, Dumitrașcu DL, Ciobanca P and Leucuta DC: Prevalence of Helicobacter pylori infection among dyspeptic patients in Northwestern Romania: A decreasing epidemiological trend in the last 30 years. Exp Ther Med 20: 3488-3492, 2020.

22. Souza RF, Bayeh L, Spechler SJ, Tambar UK and Bruick RK: A new paradigm for GERD pathogenesis. Not acid injury, but cytokine-mediated inflammation driven by HIF-2 $\alpha$ : A potential role for targeting HIF-2 $\alpha$ to prevent and treat reflux esophagitis. Curr Opin Pharmacol 37: 93-99, 2017.

23. Zhao Y, Li Y, Hu J, Wang X, Ren M, Lu G, Lu X, Zhang D and He S: The effect of Helicobacter pylori eradication in patients with gastroesophageal reflux disease: A meta-analysis of randomized controlled studies. Dig Dis 38: 261-268, 2020.

24. Bazin T, Nchare Mfondi A, Julie C, Émile JF, Raymond J and Lamarque D: Contribution of genetic amplification by PCR for the diagnosis of Helicobacter pylori infection in patients receiving proton pump inhibitors. United European Gastroenterol J 6: 1267-1273, 2018 .

25. Butt J, Varga MG, Wang T, Tsugane S, Shimazu T, Zheng W, Abnet CC, Yoo KY, Park SK, Kim J, et al: Smoking, Helicobacter pylori serology and gastric cancer risk in prospective studies from China, Japan, and Korea. Cancer Prev Res (Phila) 12: 667-674, 2019.

This work is licensed under a Creative Commons Attribution-NonCommercial-NoDerivatives 4.0 International (CC BY-NC-ND 4.0) License. 